TITLE:

\title{
Management of fetal death with placenta previa.
}

$\operatorname{AUTHOR}(\mathrm{S}):$

Taki, Mana; Sato, Yukiyasu; Kakui, Kazuyo; Tatsumi, Keiji; Fujiwara, Hiroshi; Konishi, Ikuo

\section{CITATION:}

Taki, Mana ... [et al]. Management of fetal death with placenta previa.. The journal of maternal-fetal \& neonatal medicine : the official journal of the European Association of Perinatal Medicine, the Federation of Asia and Oceania Perinatal Societies, the International Society of Perinatal Obstetricians 2012, 25(2): 196-199

ISSUE DATE:

2012-02

URL:

http://hdl.handle.net/2433/182936

\section{RIGHT:}

(c) 2012 Informa UK, Ltd.; This is not the published version. Please cite only the published version.; この論文は出版社版でありません。引用の 際には出版社版をご確認ご利用ください。 


\section{Short Report}

2 Management of fetal death with placenta previa

3

4 Mana Taki, Yukiyasu Sato*, Kazuyo Kakui, Keiji Tatsumi, Hiroshi Fujiwara, and Ikuo Konishi 5

6 Department of Gynecology and Obstetrics, Kyoto University Graduate School of Medicine 7

8 *Address correspondence and reprint requests to: Yukiyasu Sato, M.D., Ph.D.

9 Department of Gynecology and Obstetrics, Kyoto University Graduate School of Medicine, 10 Sakyo-ku, Kyoto 606-8507, Japan.

11 Tel; 81-75-751-3269: Fax; 81-75-761-3967: E-mail; yukiyasu@kuhp.kyoto-u.ac.jp 12

13 Short title; fetal death with placenta previa 
1 Abstract

2

3 Management of second- and third-trimester fetal death in the presence of placenta previa is a

4 dilemma for obstetricians. We herein describe a case of fetal death occurring at 23 weeks'

5 gestation in the presence of placenta previa. Three weeks of expectant management failed to

6 reduce uteroplacental blood perfusion evaluated with pulsatility index of the uterine artery.

7 Labor was then induced with gemeprost vaginal pessary following overnight laminaria

8 pretreatment. Vaginal delivery was achieved with total blood loss of $1900 \mathrm{ml}$. Homologous

9 blood transfusion was obviated owing to autologous blood that had been stored during the

10 waiting period.

11

12 Keywords; labor induction, laminaria, MRI, pulsatility index, expectant management 13 
1 Introduction

2

Placenta previa, which is defined as the placenta encroaching upon internal cervical os, is one of the most important causes of obstetric hemorrhage. Placenta previa persisting until the time of delivery generally requires cesarean section to prevent uncontrollable maternal hemorrhage and fetal deterioration that could be caused by partial placental abruption accompanying cervical dilation.

Fetal death can occur at any time during pregnancy. Although dilation and evacuation (D\&E) is a common practice for a second-trimester termination of pregnancy in the United States [1], this procedure is not usually performed in Japan. Moreover, in case of fetal demise, damage to the dead fetus, which is more or less unavoidable with D\&E, should be minimized for the postmortem autopsy to elucidate the cause of fetal demise. Cesarean section carries significant maternal risks especially in the second trimester without any benefit for the dead fetus [2]. In these respects, labor induction is usually selected for second- and third-trimester fetal demise in Japan.

Management of second- or third-trimester fetal death in the presence of placenta previa is a dilemma for obstetricians, since labor induction could cause intractable hemorrhage from abruption of the placenta covering the internal os. Here, we describe a case of fetal death occurring at 23 weeks' gestation in the presence of placenta previa. Labor induction after 3 weeks of expectant management brought about blood loss reaching $1900 \mathrm{ml}$. We review similar cases reported in the past literatures to discuss possible measures to reduce intrapartum blood loss in this particular condition. 
1 Case report

2

A 37-year-old primigravida, who had a history of laparoscopic excision of endometriosis, became pregnant after in vitro fertilization and embryo transfer. She consulted the clinic for small amount of painless vaginal bleeding at $23^{+0}$ weeks' gestation. Ultrasonography (USG) showed fetal cardiac arrest with the placenta completely covering the internal cervical os and she was referred to our hospital. At her first examination, vaginal bleeding had already subsided and she was hemodynamically stable with hemoglobin level of $11.9 \mathrm{~g} / \mathrm{dl}$. USG confirmed the fetal demise accompanying complete placenta previa. Magnetic resonance image (MRI) taken on the next day showed that the anterior placental edge overlapped the internal cervical os by approximately $3 \mathrm{~cm}$ with the posterior uterine wall in the retroplacental area containing adenomyosis (Figure 1A).

We chose expectant management, anticipating possible reduction of the uteroplacental blood perfusion and giving a time for autologous blood storage in preparation for hemorrhage at delivery. Maternal serum hCG, which reflects number of viable placental villi, and pulsatility index of bilateral uterine artery, which theoretically should be inversely correlated with the uteroplacental blood perfusion, were examined every 3 or 4 days. Although the maternal serum hCG level was gradually declined, pulsatility index of bilateral uterine artery was not significantly changed. Follow-up MRI taken at 14 days after the fetal demise showed significant gadolinium enhancement in the placenta (Figure 1B). Considering maternal psychological stress of harboring dead fetus for prolonged period, we decided to induce labor at 21 days after the fetal demise. No sign of maternal coagulation disorder or intrauterine infection had been noted and $800 \mathrm{ml}$ of autologous blood had been stored. The patient was thoroughly informed that emergency uterine artery embolization or hemostatic laparotomy would be required in case of intractable hemorrhage.

The day before induction, laminaria was inserted and left in the cervix overnight to obtain adequate cervical ripening. No remarkable bleeding was encountered during this preparatory step. Conforming to Japanese standard protocol, $1 \mathrm{mg}$ of gemeprost vaginal pessary (prostaglandin E1 derivative) was planned to administer every 3 hours until evacuation of the conceptus. Time course of the delivery is summarized in Figure 2. When the laminaria was removed and a first dose of gemeprost was vaginally inserted, the internal cervical os was dilated to $2 \mathrm{~cm}$. Active vaginal bleeding developed immediately afterwards with 
1 accumulative blood loss reaching $820 \mathrm{ml}$ in 1.5 hours. Iodoform-soaked gauze was packed

2 into the vagina for hemostasis. When the gauze was removed to administer a second dose of

3 gemeprost 3 hours after the first dose, active bleeding was still continued from the cervix and

4 accumulative blood loss reached nearly $1500 \mathrm{ml}$. Since the cervical dilation reached $3 \mathrm{~cm}$ and

5 the internal cervical os became in close vicinity to the anterior fetal membrane, balloon

6 catheter filled with $200 \mathrm{ml}$ of sterile water was inserted along the anterior uterine wall into the

$7 \quad$ uterine cavity. Downward traction of the balloon catheter successfully ceased the bleeding by

8 compressing detached placental surface against the posterior cervical wall (Figure 2, lower

9 rightmost illustration). Effective uterine contraction was commenced soon after

10 administration of the second dose of gemeprost. The balloon catheter was spontaneously

11 expelled from the dilated cervix, but uterine bleeding did not recur. A membrane-wrapped

12 stillborn baby (330 g) and placenta (140 g) was delivered en bloc. Total blood loss finally

13 reached $1900 \mathrm{ml}$, but homologous blood transfusion was safely obviated by autologous blood

14 transfusion. The baby was severely macerated but no external malformation was detected.

15 The parents declined to have the autopsy. Hypercoling of the umbilical cord was noted and

16 could be a primary cause of fetal demise. Her postpartum course was uneventful and she was

17 discharged 4 days after the delivery. 
Discussion

According to our pubmed search, there have been only four reported cases of second- and third-trimester fetal demise complicated with placenta previa [3, 4]. Vaginal delivery was successfully fulfilled in three of these cases at 21,23 , and 33 weeks' gestations with intrapartum blood losses of less than $500 \mathrm{ml}$. In the remaining case, cesarean section was performed at 26 weeks' gestation for history of two previous cesarean sections, leading to intraoperative blood loss of $1500 \mathrm{ml}$. These experiences suggest that vaginal delivery is a relatively safe option and cesarean section should be reserved as a last resort for second- and third-trimester fetal demise complicated with placenta previa.

In the present case, however, attempt of vaginal delivery resulted in massive hemorrhage reaching $1900 \mathrm{ml}$. The cervix was mechanically dilated with laminaria before the induction of labor and most of the hemorrhage occurred soon after its removal until placement of intrauterine balloon catheter. Vaginal packing of the gauze failed to cease the bleeding, probably because it only pushed up the whole uterus without effective compression against the detached placental surface. In contrast, placement of intrauterine balloon catheter led to complete hemostasis by compressing the detached placental surface against the cervical wall from inside the uterus. Although the balloon catheter was spontaneously expelled from the dilated cervix after effective uterine contraction was commenced, active bleeding did not recur. We attributed this to the compression subsequently exerted by the descending forebag. Therefore, cervical dilation without effective uterine contraction, which causes partial placental separation in the absence of compression by fetal membrane or fetal part, is considered to be a primary cause of excessive bleeding encountered in the present case. In fact, no mechanical cervical dilation was utilized before labor induction in the past three cases. Although cervical preparation with laminaria was reported to reduce the induction-to-delivery interval [5], we recommend that laminaria should not be used in case of placenta previa. Leaving laminaria in cervical canal until the onset of effective uterine contraction may obviate massive bleeding from the separated placenta, but this could carry high risk of uterine rupture.

Longer distance between the internal cervical os and the closest placental edge is associated with higher risk of intrapartum hemorrhage, since larger portion of the placenta needs to be separated before delivery of the fetus. In addition, the internal cervical os needs to dilate at least to the same extent as this distance in order that the fetal membrane becomes accessible 
1 through the cervix, which is prerequisite for the insertion of hemostatic balloon catheter into

2 the uterine cavity. Fortunately, placental overlap was only about $3 \mathrm{~cm}$ in the present case.

3 This enabled us to cease the placental bleeding by placement of intrauterine balloon catheter

4 without difficulty. In these respects, precise measurement of placental overlap prior to labor

5 induction is important to assess intrapartum hemorrhagic risk and to draw out hemostatic 6 strategy.

$7 \quad$ Blood supply to the placenta previa could be another important factor that determines the 8 extent of intrapartum hemorrhage. Theoretically, decrease in uteroplacental blood perfusion

9 should contribute to reduction of the blood loss during vaginal delivery. In two of the past cases as well as in the present case, expectant management for more than two weeks was introduced, anticipating possible reduction of uteroplacental blood perfusion [4]. Although the prolonged expectant management yielded favorable outcome in the past two cases, changes in the uteroplacental blood flow during the waiting period were not documented. On the contrary to our expectation, pulsatility index of the uterine artery was fluctuated and not steadily increased in the present case. Recently, Ruano et al. serially monitored uteroplacental blood flow after feticide at 29 weeks' gestation [6]. They found that the pulsatility index of uterine artery was almost doubled on the first day after the feticide. These findings suggest that the change in uteroplacental blood flow may be different between spontaneous fetal loss and feticide. Alternatively, reduction of uteroplacental blood flow may occur only in first few days after fetal demise. Indeed, in Ruano's case, no additional change in pulsatility index of uterine artery was detected for following 2 days until the delivery. Thus, considering maternal psychological stress of carrying dead fetus for extended period, we would recommend that labor induction be initiated immediately after sufficient amount of autologous blood is stored in preparation for intrapartum hemorrhage. 


\section{Declaration of Interest}

2

3 We report no declaration of interest. 
1 Reference

2

31 ACOG practice bulletin no. 102: Management of stillbirth. Obstet Gynecol $4 \quad 2009 ; 113: 748-761$.

52 Grimes DA, Schulz KF: The comparative safety of second-trimester abortion

6 methods. Ciba Found Symp 1985;115:83-101.

73 Sillender M, Krishnamurthy S: Medical management of second trimester fetal

8 death complicated by a complete placenta praevia. J Obstet Gynaecol 2000;20:537-538.

94 van der Ploeg JM, Schutte JM, Pelinck MJ, Huisjes AJ, van Roosmalen J, de

10 Vries JI: Management of fetal death after 20 weeks of gestation complicated by placenta

11 previa. J Matern Fetal Neonatal Med 2007;20:267-269.

125 Mazouni C, Vejux N, Menard JP, Bruno A, Boubli L, d'Ercole C, Bretelle F:

13 Cervical preparation with laminaria tents improves induction-to-delivery interval in

14 second- and third-trimester medical termination of pregnancy. Contraception

15 2009;80:101-104.

166 Ruano R, Kondo MM, Bunduki V, Rodeck C, Zugaib M: Follow-up of

17 uteroplacental vascularization after feticide in third-trimester therapeutic termination of

18 pregnancy with complete placenta previa. Ultrasound Obstet Gynecol 2006;27:463-465. 
$1 \quad$ Figure legend

2

3 Figure 1 T2-weighed magnetic resonance image (MRI) at 1 day after the fetal death (A) and 4 gadolinium -contrasted T1-weighed MRI at 14 days after the fetal death (B).

6 (A) Large portion of the placenta (encircled by broken line) is attached to posterior uterine wall containing adenomyosis (arrow). Note that the anterior placental edge overlaps the internal cervical os (arrowhead) by approximately $3 \mathrm{~cm}$.

(B) Size of the placenta (encircled by broken line) is reduced as compared with (A). Note that the placenta is enhanced to the same degree as the adenomyosis (arrow) in the uterine wall.

Figure 2 Time course of the induced labor at 21 days after the fetal death

Line graph of the accumulative blood loss (upper panel) shows that active bleeding began immediately after removal of the laminaria until insertion of the intrauterine balloon catheter. Overnight laminaria pretreatment caused cervical dilation and partial placental separation (lower leftmost illustration). Removal of the laminaria released the compression against separated placental edge and bleeding ensued (lower second left illustration). On the other hand, cervical dilation allowed access to the anterior fetal membrane (lower, second left illustration, arrowhead), through which balloon catheter could be inserted into the uterine cavity (lower second right illustration). Downward traction (lower rightmost illustration, closed arrow) of the balloon catheter compressed detached placental edge against the cervical wall (lower rightmost illustration, open arrow) to achieve hemostasis. 

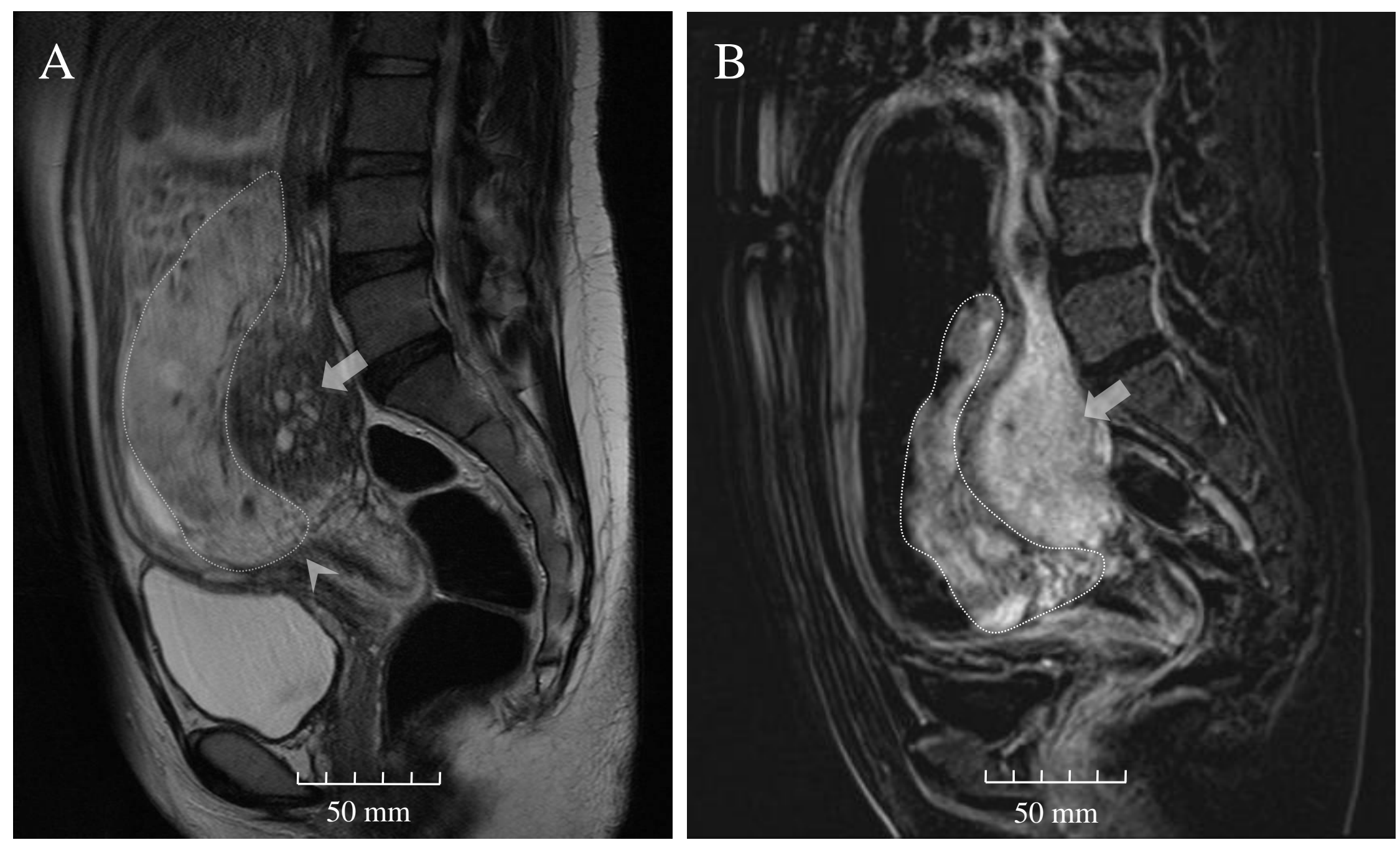

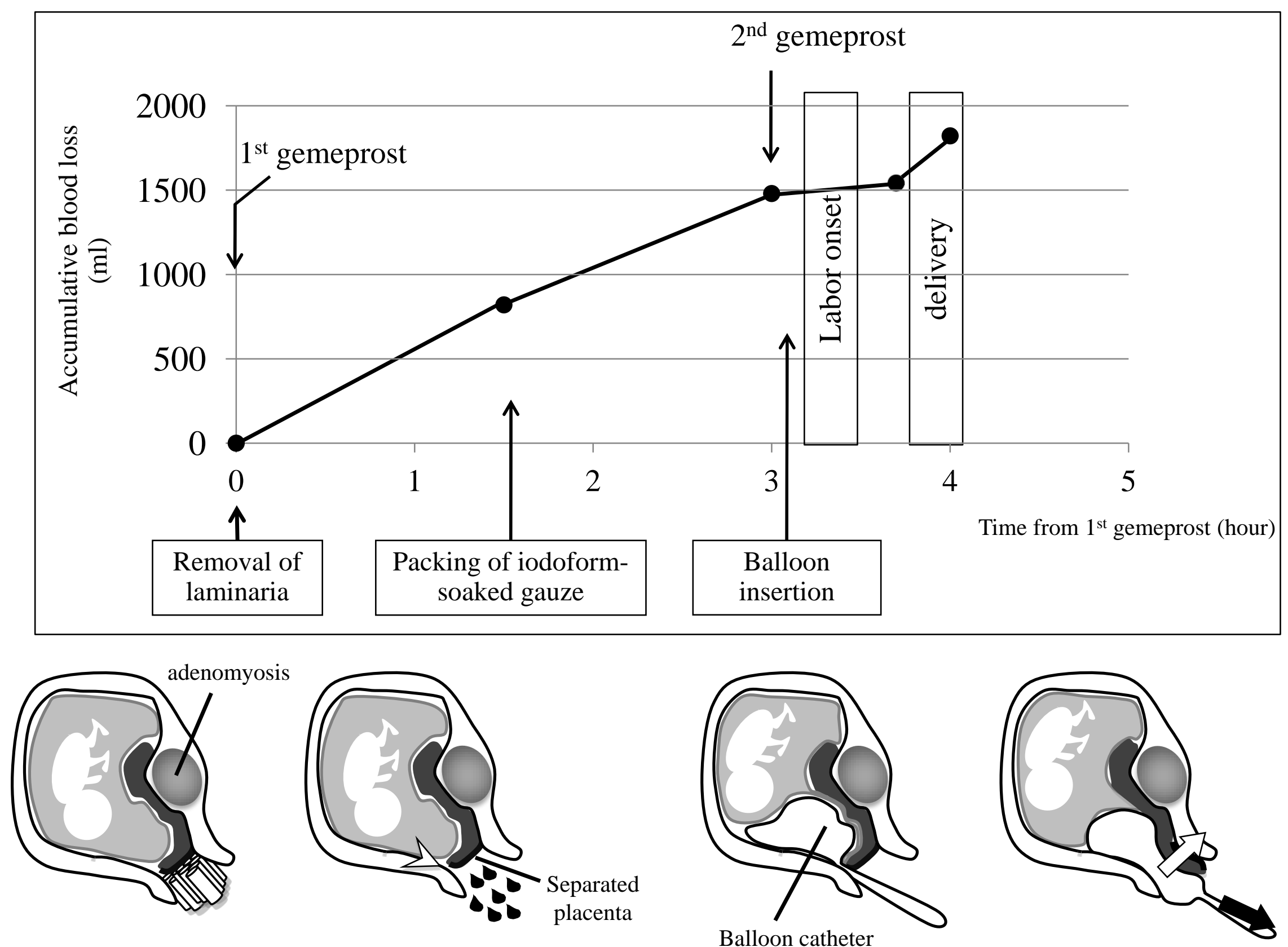